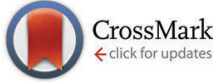

Cite this: New J. Chem., 2015, 39, 1754

Received (in Montpellier, France) 25th August 2014,

Accepted 12th December 2014

DOI: 10.1039/c4nj01430h

www.rsc.org/njc

\title{
Mesoporous silica-chondroitin sulphate hybrid nanoparticles for targeted and bio-responsive drug delivery $\dagger$
}

\author{
Krishna Radhakrishnan, ${ }^{a}$ Jasaswini Tripathy, ${ }^{a d}$ Akshay Datey, ${ }^{\text {bc }}$ \\ Dipshikha Chakravortty ${ }^{\mathrm{bc}}$ and Ashok M. Raichur*ac
}

\begin{abstract}
This work proposes the fabrication of a novel targeted drug delivery system based on mesoporous silica-biopolymer hybrids that can release drugs in response to biological stimuli present in cancer cells. The proposed system utilizes mesoporous silica nanoparticles as a carrier to host the drug molecules. A bio-polymer cap is attached onto these particles which serves the multiple functions of drug retention, targeting and bio-responsive drug release. The biopolymer chondroitin sulphate used here is a glycosaminoglycan that can specifically bind to receptors over-expressed in cancer cells. This molecule also possesses the property of disintegrating upon exposure to enzymes over-expressed in cancer cells. When these particles interact with cancer cells, the chondroitin sulphate present on the surface recognizes and attaches onto the CD44 receptors facilitating the uptake of these particles. The phagocytised particles are then exposed to the degradative enzymes, such as hyaluronidase present inside the cancer cells, which degrade the cap resulting in drug release. By utilizing a cervical cancer cell line we have demonstrated the targetability and intracellular delivery of hydrophobic drugs encapsulated in these particles. It was observed that the system was capable of enhancing the anticancer activity of the hydrophobic drug curcumin. Overall, we believe that this system might prove to be a valuable candidate for targeted and bioresponsive drug delivery.
\end{abstract}

\section{Introduction}

Bio-responsive drug delivery involves the utilization of a biologically relevant molecule or phenomenon to elicit drug release from a suitable drug carrier. These autonomous systems eliminate the need for externally manipulating the location and time of drug release. The stimuli utilized in these systems include biomolecules such as glucose, ${ }^{1,2}$ enzymes, ${ }^{3-5}$ ATP, ${ }^{6,7} \mathrm{pH}$ variations ${ }^{8-10}$ and redox potential variations. ${ }^{11,12}$

Recently, several drug delivery systems have been designed that have the ability to modulate drug release in response to these various stimuli. These systems include polymeric nanoparticles, lipid based nanoparticles, hydrogel particles and mesoporous silica nanoparticle (MSN) hybrids. ${ }^{8,13}$ Of these

\footnotetext{
${ }^{a}$ Department of Materials Engineering, Indian Institute of Science, Bangalore, 560012, India. E-mail: amr@materials.iisc.ernet.in; Fax: +9180 23600472; Tel: +918022933238

${ }^{b}$ Department of Microbiology and Cell Biology, Indian Institute of Science, Bangalore, 560012, India

${ }^{c}$ Bioengineering Program, Indian Institute of Science, Bangalore, 560012, India

${ }^{d}$ School of Applied sciences (Chemistry), KIIT University, Bhubaneswar 751024, Odisha, India

$\dagger$ Electronic supplementary information (ESI) available. See DOI: 10.1039/c4nj01430h
}

MSN-hybrids offer several advantages such as (i) the ability to retain drug molecules for prolonged time periods (ii) high drug loading efficiency due to high porosity and high specific surface area (iii) possibility for surface modification to provide additional functionalities and (iv) biochemical stability. Moreover, the mesopores of the MSN can be blocked using suitable capping agents to prevent the outward leakage of encapsulated drug molecules. Based on the properties of these capping agents, the systems may be tuned to respond to a wide array of physical, chemical or biological stimuli. ${ }^{14,15}$

Although a few MSN hybrid systems have been reported with the ability to respond to diverse bio-stimuli, such as variations in redox potentials; ${ }^{16-18} \mathrm{pH}$ variations; ${ }^{19-21}$ and enzymes, ${ }^{22-24}$ there is a lot more to be explored in this area. One important development in this field has been the introduction of capping agents that serve the dual purpose of targeting and stimuli response. An example for this kind of systems would be hyaluronic acid capped MSNs that exhibit targetability and bio-responsive drug delivery. ${ }^{18}$

In the present study, we report the fabrication of another bio-responsive MSN-hybrid system with the ability to target cancer cells. The MSN-hybrid drug delivery system has been fabricated by capping the mesopores of drug loaded MSNs with 
chondroitin sulphate (CHD). These particles display the ability to deliver hydrophobic drugs specifically into cancer cells and by utilizing the internal stimuli present there, induce bio-responsive drug release. CHD is a sulfated glycosaminoglycan that is widely distributed in the body. It is an FDA approved drug that is clinically used for treatment of arthritis and osteoporosis. In the present system we have used CHD as a biocompatible capping agent that prevents the outward diffusion of the drug molecules from the MSN mesopores, which in turn minimizes premature leakage ahead of reaching the target site. CHD also acts as a stimuli responsive component of the system as it can undergo degradation in the presence of enzymes such as lysosomal hyaluronidase present inside cancer cells. ${ }^{25,26}$ This molecule is also known to interact with cell surface receptors such as CD44 over-expressed on the surface of cancer cells. ${ }^{27}$ Although CHD has been known to possess such remarkable properties, no significant attempts have been made to utilize it as a targeted and bioresponsive platform for drug delivery. This is the first report that utilizes CHD for targeted and stimuli responsive drug delivery.

The synthesis, receptor mediated uptake and intracellular drug release of these particles are illustrated in Scheme 1. When the CHD capped MSN particles interact with cancer cells, the CHD present on the surface recognizes and attaches to the CD44 receptors facilitating the uptake of these particles. The phagocytised particles are then exposed to the degradative enzymes such as hyaluronidase present inside the cancer cells which degrades the cap resulting in drug release.

\section{Materials and methods}

$N$-Cetyltrimethyl-ammonium bromide (CTAB), $\mathrm{NaOH}$, tetraethoxysilicate (TEOS), aminopropyltrimethoxysilane (APTMS),

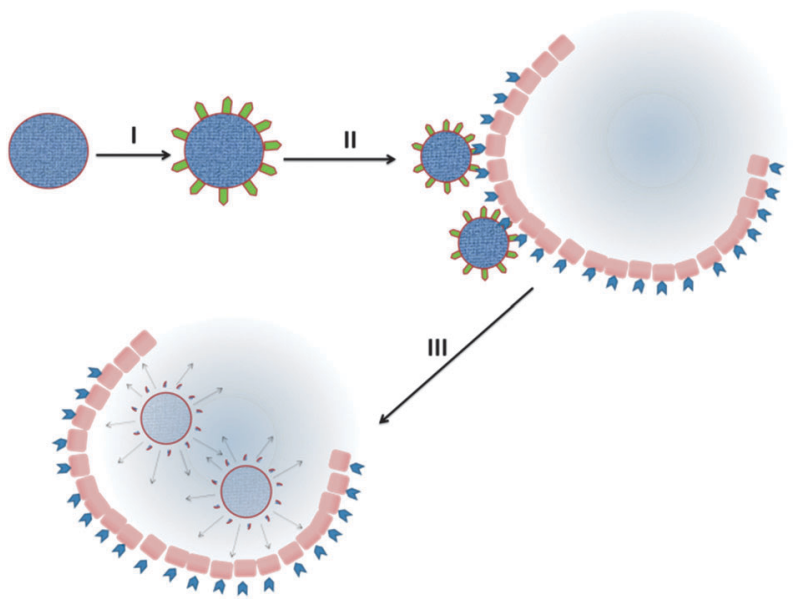

Scheme 1 General scheme illustrating drug loaded MSN-CHD nanoparticles being taken up by cancer cells and releasing the drug inside the cancer cells. (I) CHD capping on drug loaded MSN. (II) The CHD capping interacts with the CD 44 receptors overexpressed on the cancer cells resulting in efficient particle uptake. (III) Once inside the cell, the CHD cap undergoes enzymatic degradation utilizing the local biological stimuli resulting in drug release. phosphate buffer saline (PBS) 10×, CHD, 1-ethyl-3-(3-dimethylaminopropyl)carbodiimide (EDC), hyaluronidase, MTT (3-(4,5dimethylthiazol-2-yl)-2,5-diphenyltetrazolium bromide), DMEM, FCS (Fetal Calf Serum) and antibiotics were purchased from Sigma Aldrich, USA.

\section{Synthesis of $\mathrm{MSN}-\mathrm{NH}_{2}$}

Mesoporous silica nanoparticles were synthesized by modifying a previously reported procedure. Briefly, $1 \mathrm{~g}$ of CTAB in water was kept in $2 \mathrm{M} \mathrm{NaOH}$ solution and heated up to $80{ }^{\circ} \mathrm{C} .5 \mathrm{~g}$ of TEOS was added to this mixture and stirred for $2 \mathrm{~h}$. The resulting white precipitate was isolated by removing the supernatant by centrifugation at $12000 \mathrm{rpm}$. This precipitate was then washed with methanol 4 times to remove un-reacted reactants. Finally the particles were calcined at $540{ }^{\circ} \mathrm{C}$ to remove the surfactant template.

For the synthesis of amino-propyl functionalized MSNs, APTMS was used as a linker. Briefly, $200 \mathrm{mg}$ of MSN was dried at $110{ }^{\circ} \mathrm{C}$ under vacuum for $15 \mathrm{~h}$ to remove adsorbed moisture. The MSN particles were then dispersed in dry toluene (to prevent homopolycondensation of APTMS) by ultrasonication. $1 \mathrm{~mL}$ APTMS was then added to this mixture and refluxed for $20 \mathrm{~h}$ under an argon atmosphere. The resulting $\mathrm{MSN}-\mathrm{NH}_{2}$ was further purified by ultracentrifugation and washing cycles with toluene to eliminate unreacted siloxane moieties. $\mathrm{MSN}-\mathrm{NH}_{2}$ obtained was later dried at $110{ }^{\circ} \mathrm{C}$ under vacuum to remove the solvent.

\section{Attachment of CHD to MSNs}

Initially, the carboxyl groups of CHD were activated using a carbodiimide, EDC. In a typical experiment $3 \mathrm{mg} \mathrm{mL}^{-1} \mathrm{CHD}$ solution in MES buffer ( $\mathrm{pH} 5.5)$ was mixed with EDC (0.15 M) and stirred at room temperature for 2 hours. This activated the carboxyl groups of $\mathrm{CHD}$ and resulted in the formation of an $\mathrm{O}$-acyl-isourea intermediate. $50 \mathrm{mg}$ of dried $\mathrm{MSN}-\mathrm{NH}_{2}$ powder was then added to this solution and kept under constant stirring for 4 hours at ambient temperature. The activated carboxyl groups of CHD reacted readily with the amine functional groups of MSN$\mathrm{NH}_{2}$ resulting in the formation of amide bonds. The resulting MSN-CHD nanoparticles were purified by ultracentrifugation and washing cycles with double-distilled water to eliminate unreacted reactants. The purified MSN-CHD was dried overnight at $33{ }^{\circ} \mathrm{C}$ under vacuum and stored at $4{ }^{\circ} \mathrm{C}$ until further use.

\section{Material characterization}

The morphological analysis of MSN was carried out using transmission electron microscopy (TEM). Tecnai F-30 (FEI, Eindhoven, The Netherlands), equipped with a Schottky Field Emission source was used to obtain the TEM images. The samples for TEM analysis were prepared by placing a drop of the nanoparticle solution onto a copper grid.

Fourier transform infrared spectra were obtained at various stages of functionalisation on MSN, using a Thermo-Nicolet 6700 spectrometer, Thermo Scientific. The FTIR spectra of the samples pelletized using potassium bromide were recorded in the range $400 \mathrm{~cm}^{-1}$ to $4000 \mathrm{~cm}^{-1}$ with a resolution of $4 \mathrm{~cm}^{-1}$. TGA was further utilised to characterize the various functionalisation 
steps on the MSNs. STA 409PC, Netzsch at a heating rate of $10{ }^{\circ} \mathrm{C} \min ^{-1}$ was utilized to obtain the TGA spectra of the MSN samples at the various stages of functionalisation. The comparative weight loss of the sample was measured as a function of temperature, which was varied between $30{ }^{\circ} \mathrm{C}$ and $800{ }^{\circ} \mathrm{C}$ under an $\mathrm{Ar}$ atmosphere to record the TGA spectra. While the weight loss occurring before $110{ }^{\circ} \mathrm{C}$ is attributable to the loss of physically adsorbed moisture, the weight loss in the $120{ }^{\circ} \mathrm{C}$ to $750{ }^{\circ} \mathrm{C}$ regime is due to the various functional groups, organic moieties attached onto the surface of MSNs. The $\zeta$-potential variation of MSN particles was measured using a Zetasizer Nanoseries NanoZS90 equipment (Malvern Southborough, MA). The measurements were recorded by suspending the required particles in $\mathrm{pH}$ adjusted water.

\section{Drug loading and release experiments}

The drug loading was carried out prior to the capping process. In order to realize the drug loading, the $\mathrm{MSN}-\mathrm{NH}_{2}$ nanoparticles were suspended in a solution containing the required drug molecules (either $0.8 \mathrm{mg} \mathrm{mL}{ }^{-1}$ curcumin in ethanol or $0.6 \mathrm{mg} \mathrm{mL}^{-1}$ fluorouracil in $\mathrm{H}_{2} \mathrm{O}$ ). This suspension was kept for 12 hours under stirring to aid the efficient diffusion of the drug molecules into the pores of the MSN. The drug loaded nanoparticles were then centrifuged to remove un-adsorbed drug molecules. These particles were then added to a solution containing EDC activated CHD to seal the mesopores.

The drug uptake and release were calculated by measuring the absorbance of the drug molecules in the loading or release media. Absorbance measurements were carried out on a Perkin Elmer Lambda 35 spectrometer (Perkin Elmer, USA) in the wavelength range of 190 to $1100 \mathrm{~nm}$. In order to estimate the amount of unloaded drugs remaining in the drug loading solution, the absorbance of the supernatant subsequent to centrifugation of the loading solution-MSN mixture was recorded. These data, in conjunction with the data from the standard curve plotted for various concentrations of the drug, gave the amount of drug present in the supernatant after drug loading.

In a typical drug release experiment, $3 \mathrm{mg}$ of the drug loaded nanoparticles were incubated in $2 \mathrm{~mL}$ of the respective release media. In order to measure the amount of drug released, the particles were centrifuged and samples were taken from the supernatant. An equal volume of the release media was replaced to compensate for the loss due to sampling. The release curve was obtained by plotting the amount of drug released as a function of time. The total amount of drug released from MSN-CHD particles kept under probe sonication (to completely remove the $\mathrm{CHD}$ cap) for 120 minutes was taken as the $100 \%$ drug release.

\section{Cell viability assessment using an MTT assay}

HeLa cells (human cervical cancer cells) and COLO 205 (cervical cancer) were cultured in Dulbecco's Modified Medium (DMEM) and RPMI medium respectively, supplemented with $10 \%$ FBS and antibiotics. The cells were cultured in a sterile incubator at $37{ }^{\circ} \mathrm{C}, 5 \% \mathrm{CO}_{2}$. When the cells reached $90 \%$ confluence, they were harvested and seeded onto a 96 well plate at a density of $5 \times 10^{4}$ cells per $\mathrm{mL}$. Once these cells attached and flattened onto the well plate, different concentrations of the various nanoparticles were added and incubated for 24 hours. After the required incubation time, $20 \mu \mathrm{l}$ of MTT dye, a soluble yellow tetrazolium salt $\left(5 \mathrm{mg} \mathrm{ml}^{-1}\right.$ ) was added to each well and kept for another $4 \mathrm{~h}$ at $37{ }^{\circ} \mathrm{C}$. This resulted in the formation of formazan crystals which were then solubilized. The absorbance of the resulting solution was measured and the cell viability was calculated by taking the untreated cells as the control.

\section{Confocal microscopy imaging}

The CLSM images were obtained using a Carl Zeiss LSM confocal scanning system equipped with a $100 \times$ oil immersion objective with a numerical aperture of 1.4. Briefly, COLO 205 cells were seeded on a cover-slip at a cell density of $105 / \mathrm{cm}^{2}$ and incubated with curcumin loaded MSN-PRMs. After the required time period, the cells were washed with PBS and fixed using 4\% para-formaldehyde. This was followed by DAPI staining and washing with PBS. Finally, the cells were visualized under the CLSM microscope after adding the mounting media.

\section{Statistical analysis}

The Graphpad Prism software (version 5) was used for all statistical analysis. The significance of the $\zeta$-potential variations was calculated using the paired $t$ test and ANOVA. The anticancer activities of free curcumin and curcumin in MSN-CHD (at $40 \mu \mathrm{g} \mathrm{mL}^{-1}$ ) were compared using Student's $t$ test. A $P$ value of less than 0.05 was taken as significant.

\section{Results and discussion}

The MSNs were prepared by means of a surfactant templated approach. CTAB is a cationic surfactant which forms micelle structures in solution. These micelles act as templates onto which the silica species get adsorbed and later undergoes hydrolysis and condensation to form silica. The mesoporous structures of the MSNs are obtained by calcination at $540{ }^{\circ} \mathrm{C}$ which removes the surfactant template. Thus prepared MSNs with a diameter of around $170 \mathrm{~nm}$ were observed using TEM microscopy as shown in Fig. 1 . The $\mathrm{N}_{2}$ adsorption-desorption isotherms indicated a specific surface area of $781 \mathrm{~m}^{2} \mathrm{~g}^{-1}$ and average pore diameter of $2.5 \mathrm{~nm}$. The typical type IV curve observed is indicative of the mesoporous structure of these particles (ESI $\dagger$ ). The MSNs were then functionalized with amine groups using a post-synthetic grafting approach involving APTMS. Subsequent to amine functionalisation, CHD molecules were attached via carbodiimide mediated crosslinking. This resulted in the formation of an amide linkage between the amine groups of MSN and the carboxyl groups of CHD. The efficiency of the capping was expected to be further enhanced by the electrostatic interaction between the negatively charged $\mathrm{CHD}$ and the positively charged $\mathrm{MSN}-\mathrm{NH}_{2}$.

After removing the unreacted CHD molecules, the particles were characterized for successful cap attachment. Zeta potential measurements (Table 1) taken immediately after the cap attachment showed that the positive zeta potential of $\mathrm{MSN}-\mathrm{NH}_{2}$ 


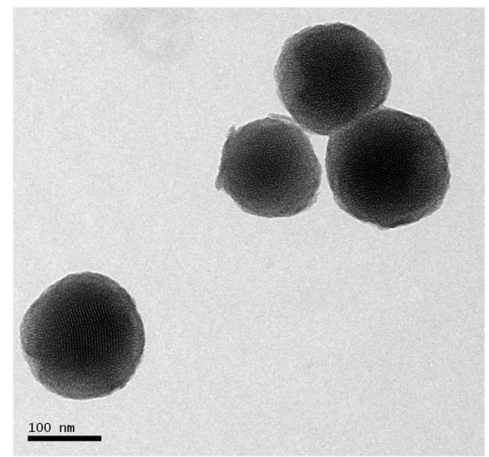

Fig. 1 TEM micrographs of the synthesized MSNs. Dilute solutions containing MSNs were coated onto a carbon grid and dried to remove the aqueous solvents.

Table 1 Zeta potential variations of MSNs suspended in water recorded at various stages of MSN-CHD fabrication

\begin{tabular}{lc}
\hline Particle & Zeta potential $^{a}(\mathrm{mV})$ \\
\hline MSN & -27.40 \\
MSN-NH & 5.03 \\
MSN-CHD & -34.16 \\
& \\
${ }^{a}$ Values represent average of three measurements. The zeta values have \\
changed significantly after each functionalisation step according to a \\
one way ANOVA test performed $(p<0.001)$.
\end{tabular}

nanoparticles had shifted from $+5.03 \mathrm{mV}$ to $-34.16 \mathrm{mV}$ due to attachment of CHD.

The formation of chemical bonds and the attachment of organic groups to the surface were characterized using TGA and IR spectroscopy. The TGA analysis carried out at different stages of functionalisation showed that there was progressive increase in weight loss after each functionalisation step (Fig. 2). This is a strong indicator for attachment of the amine groups and CHD onto the MSN surface. This was further confirmed using IR spectroscopy. The FTIR spectra of MSN samples (Fig. 3) at all stages of functionalisation showed the characteristic peak of $\mathrm{Si}-\mathrm{O}-\mathrm{Si}$ at $1087 \mathrm{~cm}^{-1}$. Upon amine functionalisation a strong peak appeared at $1580 \mathrm{~cm}^{-1}$ corresponding to the $\mathrm{NH}$ bending vibrations of the amine functional group attached to the surface. The CHD cap attachment resulted in the emergence of a new peak at $1650 \mathrm{~cm}^{-1}$ which is attributable to the $\mathrm{C}=\mathrm{O}$ stretching vibration of the carboxyl groups contributed by CHD.

CHD is a glycosaminoglycan made up of repeating units of D-glucuronic acid and $N$-acetyl-D galactosamine that are sulfated at different positions. Cells degrade CHD by using enzymes predominantly present in the lysosomes. The major enzyme involved in this process is an endo- $\beta$ - $N$-acetylhexosaminidase called hyaluronidase which is known to cleave linkages between $N$ acetylgalactosamine or $\mathrm{N}$-acetyl-galactosamine sulfate, and glucuronic acid in CHD. In order to study the bio-responsive drug release from MSN-CHD nanoparticles we have utilized this enzyme as the bio-stimulus model. Another reason for choosing hyaluronidase as the stimulus is the fact that it is widely over-expressed in the microenvironment of several cancers. ${ }^{28}$

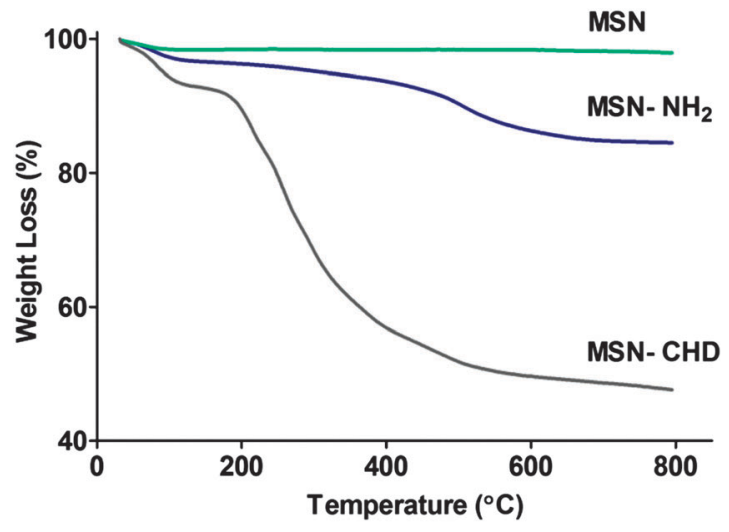

Fig. 2 TGA thermograms of the particles taken at various stages of functionalization. Samples were heated at a heating rate of $10{ }^{\circ} \mathrm{C} \mathrm{min}-1$ and the corresponding weight loss was measured. The graph displayed a progressive increase in weight loss corresponding to each functionalization step. MSNs maximum weight loss after the grafting of the bulky $\mathrm{CHD}$ groups.

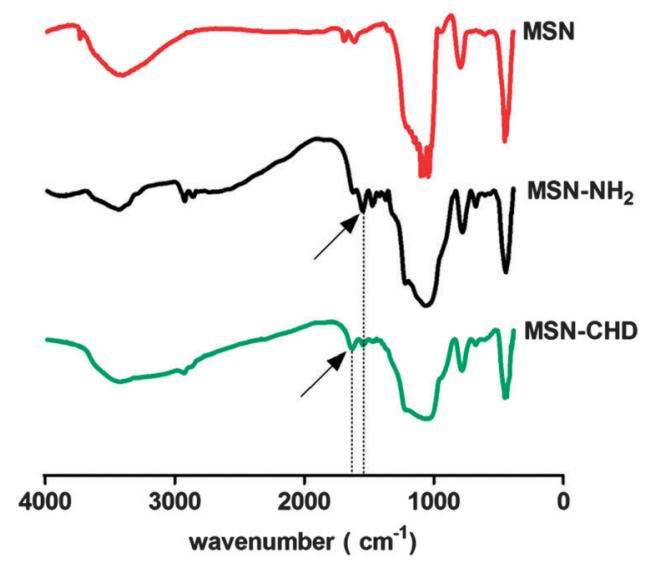

Fig. 3 FTIR spectra of $M S N, M S N-N_{2}$ and $M S N-C H D$ confirmed the attachment of the various functional molecules onto the surface of MSNs.

The drug loaded MSN-CHD nanoparticles were incubated in MES buffer at pH 5.5 in the presence of various concentrations of hyaluronidase to simulate the cancer cell microenvironment and the release was observed using absorbance spectroscopy. As a control, drug loaded MSN-CHD hybrids were incubated in PBS to simulate the physiological $\mathrm{pH}$ conditions in the absence of the bio-stimuli.

As shown in Fig. 4, the particle exhibited minimal drug leakage in the absence of the bio-stimuli. In contrast, the particles incubated in the presence of the enzyme exhibited an enhanced drug release rate. This indicates that the system can specifically induce drug release in response to biological stimuli associated with cancer cells. The drug release from the particles reached nearly $84.2 \%$ within 25 hours when incubated in media containing $500 \mathrm{U} \mathrm{mL}^{-1}$ while the particles incubated in $250 \mathrm{U} \mathrm{mL}^{-1}$ showed a lower drug release of only $56.45 \%$ within the same time period. This indicates that the drug release from the particles can be modulated by the variations in the concentration of the hyaluronidase enzyme. One possible explanation 


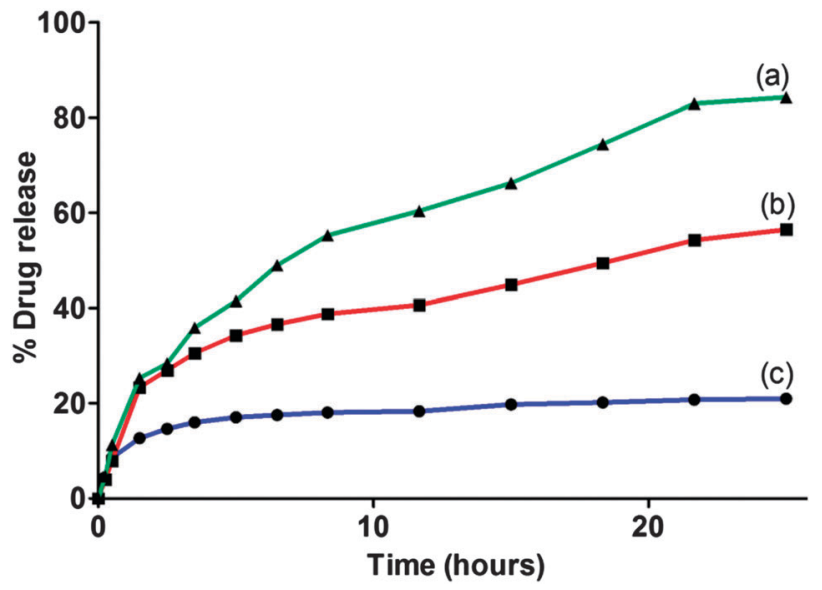

Fig. 4 Release profile from MSN-CHD in the presence of (a) $500 \mathrm{U} \mathrm{mL}^{-1}$ hyaluronidase (b) $250 \mathrm{U} \mathrm{mL}^{-1}$ hyalurondase and (c) in the absence of enzyme. The release media was either prepared in MES buffer $\mathrm{pH} 5.5$ (in the case of enzyme containing media) or in PBS buffer pH 7.4 (as in the case of control media). The concentration of drugs in the release media was evaluated using absorbance spectroscopy. The release curves indicate a concentration dependent variation in drug release rates in the presence of the triggering enzyme hyaluronidase.

for this behavior is that at lower concentrations of the enzyme the CHD cap degradation and removal may be relatively low and this partially degraded cap could be hindering the release of the drug molecules. Since the concentration of hyaluronidase I is significantly higher in certain cancer cells when compared to normal cells, this property could be useful in targeting such cancer cells. ${ }^{28}$

The cap removal process in the presence of hyaluronidase enzyme was further evaluated using zeta potential measurements. It was observed that the zeta potential of MSN-CHD which was $-34.16 \mathrm{mV}$ changed to a value of $-2.34 \mathrm{mV}$ when the particles were incubated in the presence of hyaluronidase I. This indicates that the CHD cap had been degraded into fragments by hyaluronidase enzyme and this had resulted in the reduction of the negative charge on the surface of MSNs (Fig. 5).

Next we evaluated the performance of the system in a biological scenario by using cancer cells. For this we loaded a hydrophobic anticancer drug curcumin into the pores of the particles and then observed the interactions of these particles with cancer cells. Curcumin is a photochemical drug that exhibits green fluorescence when excited with light of $420 \mathrm{~nm}$ wavelength. This property of curcumin has been utilized to study the cell interactions of the drug loaded MSN-CHD using confocal laser scanning microscopy (CLSM). The curcumin loaded MSN-CHD particles were incubated with HeLa cells and CLSM images were taken. As evidenced from the CLSM images in Fig. 6, the particles were efficiently taken up by HeLa cells. This efficiency is attributable to the high density of CD 44 receptors present on these cancer cells. CHD molecules have been reported to bind with CD 44 receptors and this could have enhanced the uptake of the nanoparticles into the cell. In order to further verify this hypothesis we blocked the CD 44 receptors on the cancer cells prior to incubation with MSN-CHD. This was carried out by using free hyaluronic acid which is another

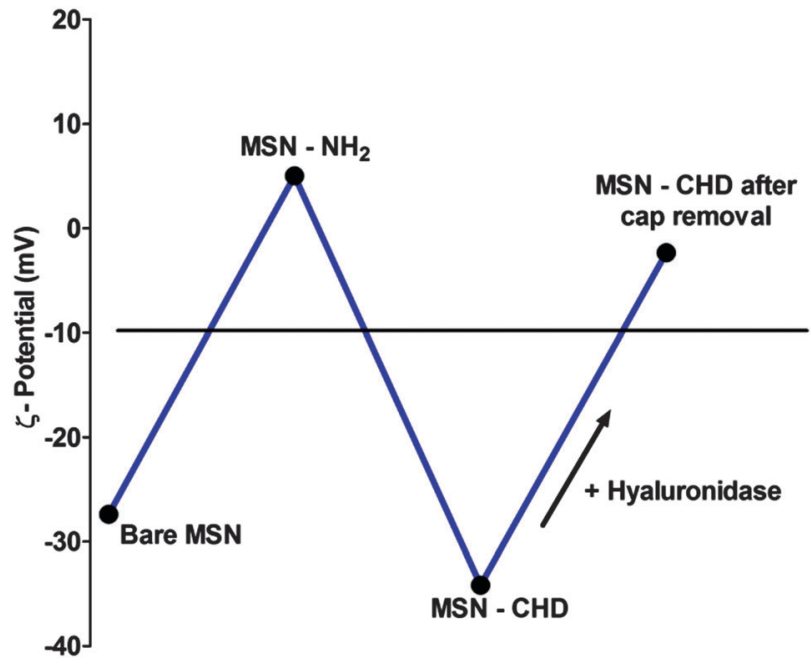

Fig. 5 Graph showing variation in the zeta potential at various stages of MSN functionalisation and CHD cap removal. The particles were suspended in water before recording the zeta potential measurements. The zeta potential fluctuated during fabrication and cap removal indicating variations in the surface chemistry of the particles at various stages.

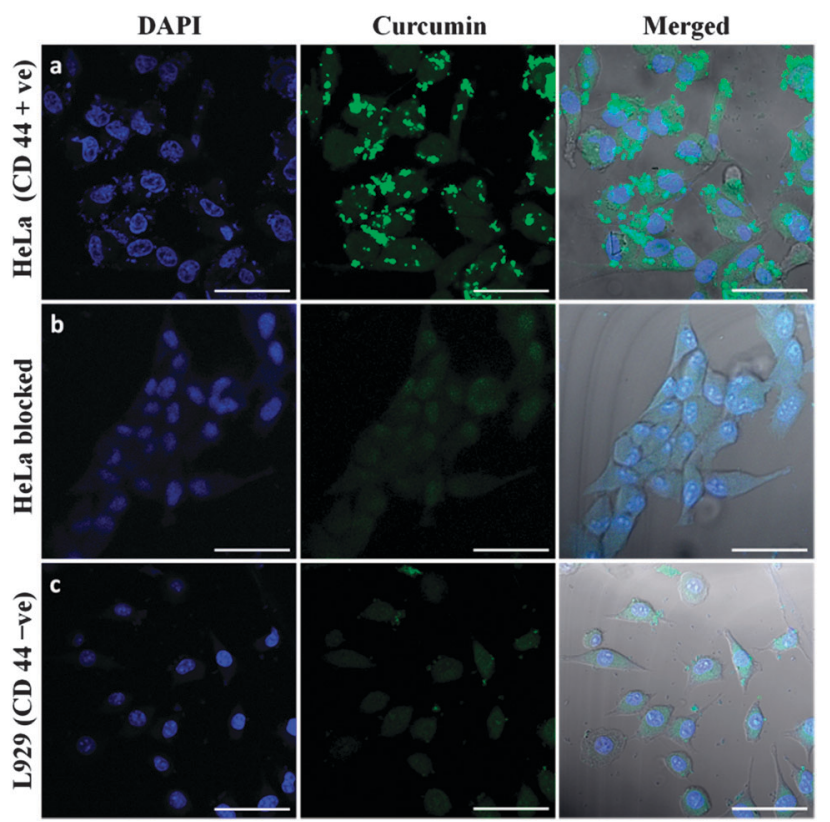

Fig. 6 CLSM images of (a) HeLa cells (b) HeLa cells whose CD44 receptors have been blocked with free HA and (c) L929 cells incubated with curcumin loaded MSN-CHD nanoparticles for 2 hours. (Scale bar $=50 \mu \mathrm{m}$ ). The CLSM images indicate that the MSN-CHD particles are taken up by cancer cells which have free unblocked HA receptors.

major ligand for this receptor. As seen in the CLSM images shown in Fig. 6, the uptake of curcumin loaded MSN-CHD was considerably reduced when the CD 44 receptors present on the cancer cell surface were blocked. This is a very good indicator that the cellular uptake of the MSN-CHD nanoparticles had taken place via the CD 44 receptors present on the cancer cells. Furthermore, CD 44 negative L929 mouse fibroblast cells were 


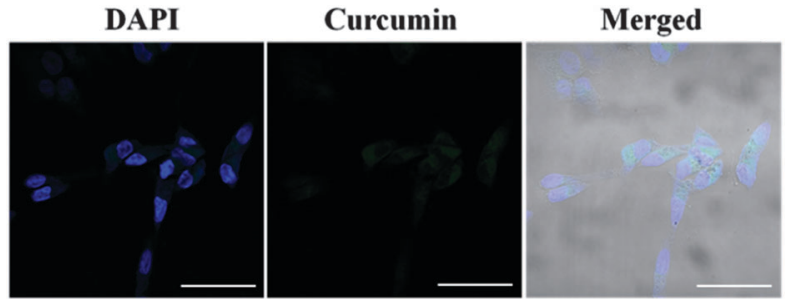

Fig. 7 CLSM images of HeLa cells incubated with free curcumin suspended in water. Due to the hydrophobic nature and poor aqueous solubility of free curcumin, it can be seen that it is not efficiently taken up by cancer cells. (Scale bar $=50 \mu \mathrm{m})$.

then used as a negative control to study the specificity of MSNCHD nanoparticles towards CD 44 positive cancer cells. HeLa and L929 cells were incubated with the same concentration of curcumin loaded MSN-CHD nanoparticles and CLSM images were taken. The CLSM images as shown in Fig. 6 indicate that the particles have preferentially accumulated inside the HeLa cells with respect to L929. While a significant green fluorescence was observed from untreated HeLa cells, only negligible fluorescence was emitted from L929. This supports the fact that the MSN-CHD particles have the ability to accumulate selectively in CD 44 positive cancer cells.

Since the loaded curcumin is a hydrophobic anticancer drug, we next studied the anticancer activity of curcumin loaded MSNs against HeLa cells. Due to its hydrophobic nature, curcumin is known to show very limited cytotoxicity in aqueous solutions. In fact, we observed that when free curcumin suspended in water was incubated with cancer cells, no significant fluorescence was observed from the cells (Fig. 7), proving that free curcumin in water is not taken up by cancer cells.

One way to overcome this limitation is to encapsulate curcumin inside suitable carriers that can be suspended in aqueous solutions. Additionally it would be beneficial if these carriers could deliver the drug directly into the interior of the cancer cells. We hypothesized that the bio-responsive MSNCHD hybrids could act as a suitable carrier for this kind of intracellular drug delivery. To demonstrate this we studied the anticancer activity of free curcumin and curcumin encapsulated in MSN-CHD against HeLa cells. As a control we have evaluated the viability of HeLa cells incubated with unloaded MSN-CHD. In order to validate any cytotoxicity contribution from the carrier, the unloaded MSN-CHD concentrations were adjusted to the corresponding concentrations of the loaded MSN-CHD studied (Fig. 8).

The results of the MTT assay carried out reveal that the curcumin encapsulated inside MSN-CHD shows more potent anticancer activity against HeLa cells when compared to free curcumin. In fact only $59.4 \%$ of the cells survived when treated with $0.1 \mu \mathrm{g} \mathrm{mL} \mathrm{mL}^{-1}$ curcumin encapsulated inside MSN-PRM as opposed to $97.94 \%$ of the cells surviving after treatment with free curcumin in water $(p<0.05)$. More significant difference in anticancer activity was observed at higher concentrations of curcumin with the percentage of cell death being $69.87 \%$ for curcumin in MSN-PRM $\left(40 \mu \mathrm{g} \mathrm{mL}^{-1}\right)$ and $19.6 \%$ for free

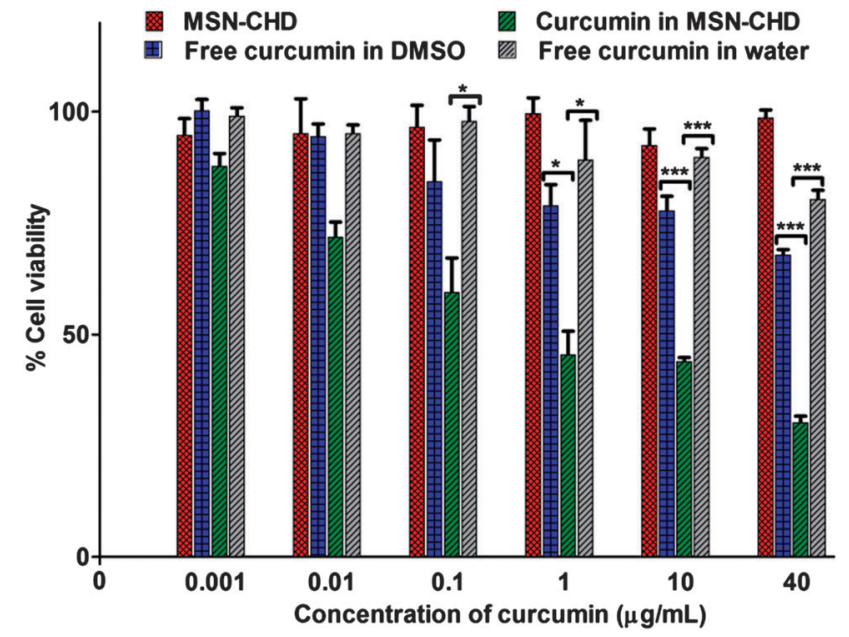

Fig. 8 Results of the MTT assay showing the anticancer activity of curcumin encapsulated inside MSN-CHD when compared to free curcumin in DMSO or in water. The error bar represents the standard deviation of three measurements. The readings that are significantly different according to a one way ANOVA test have been marked in the graph $\left({ }^{* *} p<0.0001,{ }^{*} p<0.05\right)$.

curcumin in water $\left(40 \mu \mathrm{g} \mathrm{mL} \mathrm{mL}^{-1}\right)(p<0.0001)$. Interestingly, the anticancer activity of curcumin in MSN-CHD was significantly higher than curcumin dissolved in DMSO as well. The difference was significant at higher concentrations with the cell death being around $32.18 \%$ at $40 \mu \mathrm{g} \mathrm{mL} \mathrm{m}^{-1}$ of curcumin $(p<0.0001)$. This may be due to the enhanced ability of curcumin in MSN-CHD to accumulate inside HeLa cells as opposed to free curcumin. We also observed that the unloaded MSN-CHD particles exhibited no significant cytotoxicity within the range of concentrations tested.

\section{Conclusion}

In summary we have reported the fabrication of a novel bioresponsive and targeted drug delivery system which utilizes mesoporous silica nanoparticles as the drug carrier and chondroitin sulphate as the degradable cap. The cap in addition to being biodegradable, provides targetability to the particles towards CD 44 overexpressing cells. In the proof of concept studies, we have demonstrated that these particles can release drug molecules in the presence of the enzyme stimulus hyaluronidase and that the particles can deliver hydrophobic anticancer drugs specifically to CD 44 overexpressing cancer cells. The particles were also seen to enhance the anticancer activity of the hydrophobic drug curcumin. These findings clearly indicate that the mesoporous silica nanoparticle-chondroitin sulphate system is a highly promising candidate for bioresponsive and targeted drug delivery.

\section{Notes and references}

1 K. Ishihara and K. Matsui, J. Polym. Sci., Part C: Polym. Lett., 1986, 24, 413-417.

2 Y. Zhao, B. G. Trewyn, I. I. Slowing and V. S.-Y. Lin, J. Am. Chem. Soc., 2009, 131, 8398-8400. 
3 R. V. Ulijn, N. Bibi, V. Jayawarna, P. D. Thornton, S. J. Todd, R. J. Mart, A. M. Smith and J. E. Gough, Mater. Today, 2007, 10, 40-48.

4 K. Radhakrishnan and A. M. Raichur, Chem. Commun., 2012, 48, 2307-2309.

5 H. Li, S. S. Yu, M. Miteva, C. E. Nelson, T. Werfel, T. D. Giorgio and C. L. Duvall, Adv. Funct. Mater., 2013, 23, 3040-3052.

6 S. Biswas, K. Kinbara, T. Niwa, H. Taguchi, N. Ishii, S. Watanabe, K. Miyata, K. Kataoka and T. Aida, Nat. Chem., 2013, 5, 613-620.

7 V. C. Özalp and T. Schäfer, Chem. - Eur. J., 2011, 17, 9893-9896.

8 A. E. Felber, M.-H. Dufresne and J.-C. Leroux, Adv. Drug Delivery Rev., 2012, 64, 979-992.

9 Y. Qiu and K. Park, Adv. Drug Delivery Rev., 2012, 64(suppl), 49-60.

10 Q. Yin, J. Shen, Z. Zhang, H. Yu and Y. Li, Adv. Drug Delivery Rev., 2013, 65, 1699-1715.

11 N. Graf and S. J. Lippard, Adv. Drug Delivery Rev., 2012, 64, 993-1004.

12 Y.-J. Pan, Y.-Y. Chen, D.-R. Wang, C. Wei, J. Guo, D.-R. Lu, C.-C. Chu and C.-C. Wang, Biomaterials, 2012, 33, 6570-6579.

13 R. de la Rica, D. Aili and M. M. Stevens, Adv. Drug Delivery Rev., 2012, 64, 967-978.

14 I. I. Slowing, J. L. Vivero-Escoto, C.-W. Wu and V. S.-Y. Lin, Adv. Drug Delivery Rev., 2008, 60, 1278-1288.

15 B. G. Trewyn, S. Giri, I. I. Slowing and V. S.-Y. Lin, Chem. Commun., 2007, 3236-3245.
16 Z. Luo, K. Cai, Y. Hu, L. Zhao, P. Liu, L. Duan and W. Yang, Angew. Chem., Int. Ed., 2011, 50, 640-643.

17 Q. Zhang, F. Liu, K. T. Nguyen, X. Ma, X. Wang, B. Xing and Y. Zhao, Adv. Funct. Mater., 2012, 22, 5144-5156.

18 B. Chang, D. Chen, Y. Wang, Y. Chen, Y. Jiao, X. Sha and W. Yang, Chem. Mater., 2013, 25, 574-585.

19 D. Tarn, M. Xue and J. I. Zink, Inorg. Chem., 2013, 52, 2044-2049. 20 Q. He, Y. Gao, L. Zhang, Z. Zhang, F. Gao, X. Ji, Y. Li and J. Shi, Biomaterials, 2011, 32, 7711-7720.

21 C.-H. Lee, S.-H. Cheng, I.-P. Huang, J. S. Souris, C.-S. Yang, C.-Y. Mou and L.-W. Lo, Angew. Chem., Int. Ed., 2010, 49, 8214-8219.

22 C. Coll, L. Mondragón, R. Martínez Máñez, F. Sancenón, M. D. Marcos, J. Soto, P. Amorós and E. Pérez Payá, Angew. Chem., Int. Ed., 2011, 50, 2138-2140.

23 A. Bernardos, E. Aznar, M. D. Marcos, R. Martínez Máñez, F. Sancenón, J. Soto, J. M. Barat and P. Amorós, Angew. Chem., Int. Ed., 2009, 48, 5884-5887.

24 A. Schlossbauer, J. Kecht and T. Bein, Angew. Chem., Int. Ed., 2009, 48, 3092-3095.

25 T. Honda, T. Kaneiwa, S. Mizumoto, K. Sugahara and S. Yamada, Biomolecules, 2012, 2, 549-563.

26 R. Stern and M. J. Jedrzejas, Chem. Rev., 2006, 106, 818-839.

27 E. N. Harris and P. H. Weigel, in Animal Lectins: A Functional View, ed. G. R. Vasta and H. Ahmed, CRC Press, Boca Raton, 2009, pp. 171-192.

28 Z. Chen, Z. Li, Y. Lin, M. Yin, J. Ren and X. Qu, Chem. - Eur. J., 2013, 19, 1778-1783. 